\title{
EDITORIAL
}

\section{Special Issue: Self-Assembled Materials}

Polymer Journal (2012) 44, 451; doi:10.1038/pj.2012.62

$\mathrm{R}^{\mathrm{c}}$ ecently, polymers as soft materials have attracted a great deal of attention as functional materials in the fields of electronic, optical, photochemical and energy devices, and for use as environmental materials and in bio-applications. The highly favorable low energy-consumption processing, environmental benignancy, flexible structures and biocompatibility are characteristics of these polymeric materials. For applications of polymers as functional materials, a tight control of the assembled structures of polymers is very important as this will determine their properties. Induction of microphase separated structures and liquid crystallinity in bulk, and the formation of gels and micelles in solution are representative approaches to the achievement of targeted functions. To obtain such controlled assembled structures, synthetic polymers with precise structures and biopolymers, such as DNA and peptides, have been used because of the advancements in polymer synthesis and bio-related chemistry, respectively.

This special issue, 'Self-Assembled Materials' covers a variety of self-assembled materials from polymers to polymer/inorganic hybrids, assemblies of low molecular weight compounds and nanoparticles. Interdisciplinary research between polymers and other materials is expected to uncover new science and innovative frontiers in the field of polymeric and self-assembled materials. For example, supramolecular chemistry has also been an emerging area over the past two decades. Use of noncovalent interactions, such as hydrogen bonding, ionic interactions and charge transfer interactions, has now become a powerful tool in constructing molecular assemblies with controlled structures in the fields of polymer science and supramolecular chemistry. The construction of hybrids of polymers and inorganic materials or metal particles is also a growing area. Enhanced or new functions are expected for these hybrid materials.

This special issue collects papers covering a wide variety of self-assemblies of polymers and polymer-related materials. As guest editors of this thematic issue, we hope that this collection of papers will stimulate further progress in material science as well as polymer science. Finally, the editors thank all authors and referees for their contribution to this issue.

Takashi Kato ${ }^{1}$, Sadahito Aoshima ${ }^{2}$ and Hirotsugu Kikuchi ${ }^{3}$ ${ }^{1}$ Department of Chemistry and Biotechnology, School of Engineering, The University of Tokyo, Tokyo, Japan; ${ }^{2}$ Department of Macromolecular Science, Graduate School of Science, Osaka University, Osaka, Japan and ${ }^{3}$ Institute for Materials Chemistry and Engineering, Kyushu University, Fukuoka, Japan E-mail: kato@chiral.t.u-tokyo.ac.jp 